\title{
Crítica de las ideologías y comprensión de sí. Aportes hermenéuticos.
}

Mariana Leconte

\section{Introducción}

Nos proponemos en este ensayo indagar en qué medida la crítica de las ideologías asume en sus pretensiones una de las principales conquistas de la racionalidad postmetafísica, a saber, el reconocimiento de la finitud del pensamiento y la existencia humana en general, particularmente en un aspecto específico: la propia situación, pertenencia o ser-en-el-mundo.

En el fondo, la cuestión nos conduce a indagar a la vez los límites de la crítica de las ideologías y a preguntarnos sobre su necesidad de reconocimiento y diálogo con las pretensiones de la hermenéutica filosófica.

Como constitutivas de un cierto trasfondo común orientado hacia una nueva racionalidad en nuestra época, podemos reconocer, tanto en la crítica de las ideologías como en la hermenéutica filosófica, una opción frente a la noción restringida de experiencia y lenguaje propias del positivismo -que reducen la racionalidad a racionalidad científico-técnica-, y una apuesta por el carácter situado de la razón, que renuncia a las pretensiones de totalización y homogeneización propias de una larga tradición en Occidente, desde el reconocimiento de la historicidad del sujeto y desde una conciencia clara del papel del lenguaje en el desarrollo racional.

Sin perder de vista ese trasfondo común, intentaremos dilucidar un límite de la propuesta de la crítica de las ideologías respecto de esa asunción de la finitud de la existencia humana, y ver en ese límite una posibilidad de apertura a la hermenéutica y una exigencia de reconocimiento de sus pretensiones.

Para ello, comenzaremos nuestro desarrollo con una síntesis de algunos aspectos de la teoría habermasiana, para abordar en el segundo parágrafo algunos desarrollos de la hermenéutica de Paul Ricoeur que nos permitirán desplegar en las conclusiones nuestras intuiciones respecto de la cuestión planteada.

\section{Desarrollo}

\subsection{Algunos aspectos relevantes de la filosofía habermasiana}

La propuesta de Habermas se recorta sobre un intento de fundamentación epistemológica de las ciencias sociales como teorías. Esta fundamentación busca una alternativa tanto frente al absolutismo de la filosofía trascendental como frente al idealismo de la filosofía reflexiva de Hegel y Husserl, y al materialismo de supuestos objetivistas de la crítica social marxista.

Sin embargo, a pesar de esto último, la teoría habermasiana se inserta en la tradición de la filosofía marxista e intenta reformular, rescatándolo, su sentido crítico, a la luz de las nuevas circunstancias sociales y económicas, y contando con una concepción más amplia de la racionalidad.

Su propuesta busca integrar el planteo trascendental con un planteamiento evolucionista. Se trata de superar el objetivismo de las ciencias que niegan la participación subjetiva en la constitución de los objetos de la experiencia posible, a la vez que el monologismo de la filosofía trascendental kantiana o de la filosofía de la conciencia, que

\footnotetext{
* Correo electrónico: marianaleconte@hotmail.com 03783 - 429957
} 
hacen depender la síntesis del objeto de un sujeto trascendental o absoluto. Habermas atribuye la síntesis objetiva a la "operación a la vez empírica y trascendental de un sujeto-especie que se genera históricamente” (Conocimiento e Interés) ${ }^{1}$.

Desde esta perspectiva se comprende la imposibilidad de comprender el mundo en que vivimos y de construir el mundo que queremos a partir de la actitud monológica y observadora del científico. En la medida en que nuestra experiencia y nuestro lenguaje -nuestro mundo, en suma- se constituyen intersubjetivamente, sólo participando de procesos de entendimiento se vuelve accesible al científico social la comprensión de sentido.

Ya Conocimiento e Interés realiza una apuesta fuerte contra el reduccionismo del objetivismo sociológico y la teoría empirista de la ciencia, que reducen la racionalidad al ámbito de las acciones instrumentales. Con la teoría de los intereses cognoscitivos realiza Habermas una distinción fundamental entre la constelación lenguaje-acciónexperiencia en la actividad instrumental y la actividad comunicativa respectivamente, que abre paso al concepto de racionalidad comunicativa y hace frente al peligro de identificar el proceso de constitución del objeto sensible con que opera la ciencia empírica y el mundo simbólicamente pre-estructurado con que se enfrenta el científico social.

En tanto las ciencias sociales y las ciencias del espíritu se encuentran desde su base ligadas a la práctica social lingüísticamente pre-estructurada, su fundamentación dirige la reflexión necesariamente a la condición de posibilidad de una forma comunicativa de vida.

La teoría de la acción comunicativa viene a responder, así, a tres cuestiones: la necesidad de un concepto de racionalidad comunicativa que haga frente al reduccionismo cientificista, la necesidad de un concepto de sociedad que integre el nivel sistemático y el nivel del mundo de la vida, y, una teoría de la sociedad que, en coherencia con los conceptos anteriormente nombrados, interprete la problemática actual en clave de absorción de los ámbitos de acción comunicativa en los sistemas de acción organizados y autonomizados según una racionalidad instrumental.

En este sentido, la teoría de la acción comunicativa asume, por un lado, una pretensión crítica de poner al descubierto la nueva forma de ideología, a saber, la instrumentalización de ámbitos en principio referidos a la comunicación, y de reconstruir las distorsiones y restaurar la comunicación quebrada; y, por otro lado, una pretensión "escatológica” de realizar en la historia una vida social regulada por acuerdos intersubjetivamente consensuados, fundados en la posibilidad de una comunidad de comunicación.

En esta dirección se comprende el cambio de paradigma que la filosofía del giro lingüístico permitió realizar a la teoría habermasiana. Con los aportes de la filosofía del lenguaje, pragmáticamente reconsiderada, la teoría habermasiana puede fundamentarse indagando las condiciones de posibilidad del actuar comunicacional en el telos intersubjetivo o dialógico inherente al lenguaje. La teoría de la acción comunicativa, en efecto, "se entiende como reconstrucción hipotética del saber preteórico que los sujetos capaces de lenguaje y de acción inevitablemente aplican cuando, y en la medida en que, participan competentemente de interacciones mediadas lingüísticamente” ${ }^{2}$.

Frente a la hermenéutica, que supone este acuerdo intersubjetivo como dado de modo aproblemático, la teoría habermasiana acentúa el carácter crítico necesario para reconocer las distorsiones ideológicas de la comunicación, debidas a la violencia disi-

\footnotetext{
${ }^{1}$ Cfr. Jürgen Habermas, Conocimiento e Interés, Madrid, Edit. Taurus, 1987.

${ }^{2}$ Cfr. Jürgen Habermas, Lógica de las ciencias sociales, Madrid, Tecnos, 1988, p.494.
} 
mulada, y supone la comunidad de comunicación como idea reguladora, que dirige los esfuerzos humanos hacia el futuro.

La progresiva racionalización del mundo -entendido en Habermas como mundo de la vida en sentido fenomenológico- deberá entenderse como progresiva conquista o despliegue de lo que es ya, sin embargo, inherente a todo acto de habla: la posibilidad de entendimiento intersubjetivo, de consenso sin coacción.

En este sentido, cobra protagonismo la racionalidad de los procedimientos frente a la racionalidad sustantiva de los contenidos. No se considera racional el mundo de la vida en que nos hallamos instalados por el sólo hecho de ser lo vigente, ni el estado de cosas con que damos en el mundo objetivo, ni el mundo subjetivo que proyectamos, sino "la solución de problemas que logramos en nuestro trato con la realidad, atenido a procedimientos” (Pensamiento post-metafísico, p.45).

La opción por esta racionalidad procedimental implica el reconocimiento de la imagen del mundo a la que pertenecemos como una imagen del mundo, es decir, implica la posibilidad de reflexionar sobre la propia imagen del mundo en cuanto tal, de distinguir nuestra propia proyección de mundo, del estado de cosas, y del mundo de las relaciones intersubjetivas. Implica, pues, una valorización del paso del mhytos al logos. Esta valorización se correlaciona con la posibilidad de descentración de sí y de la propia visión del mundo.

Sintetizando, la racionalidad comunicativa implica una ampliación de la racionalidad cognitivo-instrumental, y de este modo, posibilita una comprensión de los procesos sociales en una nueva clave, capaz de dar cuenta de las nuevas ideologías y violencias que afectan la actualidad de la vida social. En este sentido, reivindica la pretensión crítica como teoría de la sociedad.

\subsection{Finitud y Crítica de las ideologías}

Intentaremos poner de manifiesto uno de los aspectos implicados en el reconocimiento del carácter finito de la existencia humana, a saber, el de pertenencia o ser en el mundo, para poder dilucidar la cuestión que hemos puesto en primer plano en este ensayo, y arribar a algunas conclusiones.

En efecto, en primer lugar, el reconocimiento del carácter finito de la existencia humana implica el reconocimiento de su carácter de situado, encarnado, histórico, temporal, y por tanto, la renuncia a la omnipotencia de su punto de vista auto referencial, a su pretensión de ocupar el lugar del fundamento, y al solipsismo.

Desde esa perspectiva, tiene sentido la afirmación hermenéutica que considera imposible una crítica exhaustiva de la historia, en tanto nosotros mismos estamos situados en ella. No existe, efectivamente, un punto cero desde el cual esta crítica pueda ser hecha. De esto se deriva la exigencia de una comprensión y una desapropiación de sí cual la que se da en la hermenéutica de los textos poéticos, por ejemplo- que provea al sujeto de la interpretación de la situación propia, como precondición para su comprensión del otro ${ }^{3}$.

Heidegger dio cuenta en Ser y Tiempo de esta situación de todo Dasein que Gadamer llamó pertenencia. En tanto somos, somos siempre ya en un mundo. No somos en ningún momento sujetos sin mundo. El ser-en constituye fundamentalmente el ser de nuestra existencia, de modo que tanto la problemática de la objetividad como la de la

\footnotetext{
${ }^{3}$ Lo de precondición no debería entenderse sólo en sentido temporal, dado que, con Levinas, podríamos considerar la desapropiación de sí -y la comprensión de la propia situación- a partir del advenimiento del otro, que quiebra la seguridad del "propio lugar bajo el sol”.
} 
subjetividad presuponen una relación de inclusión anterior "que engloba al sujeto presuntamente autónomo y al objeto presuntamente adverso" $"$.

Desde esta perspectiva, y en tanto, además estamos "bañados" por el lenguaje, todo diálogo que iniciamos se inserta ya sobre un diálogo que nos precede, al que alcanzamos ya iniciado. Por ello, el lugar de la interpretación no es tanto el diálogo sino la tradición que lo engloba.

En su artículo "Hermenéutica y crítica de las ideologías" ${ }^{5}$ propone Ricoeur como modelo de reconsideración de la pretensión hermenéutica desde su propio seno, la hermenéutica del texto. El texto no es para Ricoeur meramente un caso particular de comunicación humana sino "el paradigma de la distanciación en la comunicación". Por ello, en la hermenéutica del texto queda de manifiesto la necesidad de un rodeo por la explicación y la comprensión para la interpretación. En efecto, la distanciación presupuesto de la crítica- es inherente al carácter de mediación del texto. El texto se independiza del autor, sus intenciones y su contexto. El texto se distancia de su autor como se distancia de su primer destinatario. Desde esta distancia emerge lo que verdaderamente debe ser comprendido en un texto: el mundo del texto. El mundo del texto no es lo que es dicho sino "aquello acerca de lo cual se dice" ${ }^{6}$. El texto -y nos referimos ahora al texto escrito específicamente- se refiere a un mundo, pero esta referencia no es una referencia meramente descriptiva como la del discurso científico. Es precisamente desde las ruinas del discurso descriptivo desde donde la función poética hace surgir una pertenencia "a un orden de cosas que precede nuestra capacidad de oponer estas cosas como objetos que enfrentan a un sujeto" ${ }^{7}$. Esto es, hace surgir nuestro ser en el mundo. Ricoeur define esta referencia poética como ficción y redescripción, lo que en términos aristotélicos llamaríamos mythos que es camino a la mímesis, no en tanto imitación sino en tanto metamorfosis o redescripción. El texto, en su supresión de la referencia a los entes como objetos de nuestra manipulación, abre "el mundo de nuestro arraigo originario". Así, desmiente la pretensión de la conciencia de fundar toda significación desde sí, y la abre a la posibilidad de comprenderse desde un sí mismo más vasto abierto por el mundo propuesto por el texto, esto es, de comprenderse desde un más allá de sí. Interpretar es, aquí, "explicitar la forma de ser-en-el-mundo desplegada ante el texto" 8

En este punto puede verse la función crítica de la hermenéutica del texto. En efecto, comprender el texto no es proyectarse en él sino exponerse a él. Y en esta exposición, extraer de la apropiación de la propuesta de mundo que el texto abre un sí mismo más vasto, más rico. La ficción, la referencia poética del texto, posibilita la redescripción del propio yo, posibilita "variaciones imaginarias" del propio ego. Esta posibilidad abierta por el texto es un camino fundamental para la crítica de las ilusiones del sujeto. Podría incluso hasta hacerse una analogía con la función terapéutica del psicoanálisis. En la relación psicoanalista-paciente, hay un cierto mythos recreado, se ponen en juego

\footnotetext{
${ }^{4}$ Cfr. Javier Bengoa Ruiz de Azúa, De Heidegger a Habermas, Hermenéutica y fundamentación última en la filosofía contemporánea, Barcelona, Herder, 1992, p.144.

${ }^{5}$ Paul Ricoeur, "Hermenéutica y Crítica de las ideologías", en Hermenéutica y Acción, Buenos Aires, Docencia, 1988 (2da edic.), pp. 183 - 222.

${ }^{6}$ Cfr. Paul Ricoeur, "Hermenéutica de la idea de Revelación", en Fe y Filosofía, Problemas del lenguaje religioso, Buenos Aires, Docencia-Almagesto, 1994, p. 182.

${ }^{7}$ Ibid, p.183.

${ }^{8}$ Paul Ricoeur, "La fonction herméneutique de la distantiation”, Philosophy Today, XVII, Citado según su reproducción en Paul Ricoeur, Du texte á la action, Essais d’herméneutique II, París, Seuil, 1998, pp.101-117.
} 
las escenas primitivas y el paciente tiene la posibilidad de redescribir, de transformar en esa mímesis algunas identificaciones.

En suma, no hay posibilidad para la conciencia de auto - reflexión absoluta: en primer lugar, el cogito está siempre mediatizado por signos, se objetiva a través de obras, instituciones, representaciones. No dispone de sí de manera inmediata. Reclama también interpretación. En segundo lugar, el sujeto autónomo apartado del objeto es una abstracción de una relación anterior que incluye objeto y sujeto: su pertenencia a un mundo. La reflexión es así, segunda respecto de este estar-ya-ahí. Es una distancia crítica que se recorta sobre la previa pertenencia, sobre la inclusión en el seno de una experiencia $^{9}$.

Es necesaria, pues, una hermenéutica para comprenderse a sí mismo, o mejor aún, una dialéctica entre hermenéutica y reflexión. Esto significa para nuestra cultura, donde los signos son obras y textos, la necesidad de la apropiación. En todo caso, la apropiación por vía hermenéutica deberá ser una apropiación crítica, que recree la cultura y la despoje de los elementos ideologizados. Pero sólo podrá hacerse desde un círculo que vaya de la comprensión de la propia situación a la crítica y de la crítica a la comprensión de la propia situación.

Finalmente, en estos análisis de Ricoeur está presente la idea de la verdad como manifestación: no se trata de verificar lo objetivado por la conciencia, sino de dejarse dar lo que se muestra. Esta instancia de alteridad constituida por el texto poético, entre otras, enfrenta al Dasein al sin-sentido de su pretensión representativa total, arrojada a la cual, esa alteridad quedaría reducida a Nada. La pretensión verificadora y la pretensión de autonomía de la conciencia, dejan en lo oculto la pertenencia de la conciencia y del texto a un mundo, cierran la posibilidad de la desposesión de la conciencia, y por tanto, la mantienen en el engaño de su auto-fundación. Por el contrario, Ricoeur pone de manifiesto de qué manera el texto poético abre para la conciencia una propuesta de mundo habitable que no se reduce a lo por ella puesto, que la abre a una comprensión de sí sin origen en sí, y la despierta a la secundariedad de su reflexión respecto de su pertenencia a un mundo. La convoca, así, a una interpretación infinita y, haciéndolo, la despierta del engaño.

\section{Conclusiones}

Intentaremos ahora indagar en qué medida la finitud de la existencia humana, en este aspecto específico referido en el parágrafo anterior, está asumida en los planteos habermasianos. Esta asunción podría ser un fuerte punto de encuentro con la hermenéutica filosófica.

No creemos que esta afirmación del sujeto como finito esté, en general, ausente en la obra de Habermas. Muy por el contrario, él mismo da cuenta en su propia producción de los caracteres del pasaje del pensamiento metafísico al postmetafísico, en cuyo trasfondo late el descubrimiento de la temporalidad y la contingencia de la existencia humana.

Este reconocimiento es asumido por Habermas, en primer lugar, en la opción por una racionalidad procedimental frente a la racionalidad sustantiva de la metafísica. Esta opción supone la caída de la pretensión del “conocimiento del todo” que la filosofía sos-

\footnotetext{
${ }^{9}$ Lo que Heidegger llama "facticidad".
} 
tuvo por siglos, además de la apertura a la necesidad del diálogo intersubjetivo para nuestras aproximaciones a la verdad.

El planteo de una razón finita se evidencia especialmente en el giro lingüístico de su filosofía, que determina en ella un cambio de paradigma, un tránsito desde la filosofía de la reflexión y la conciencia a una teoría del actuar comunicacional. Esto es, desde una filosofía que fundamenta sus pretensiones monológicamente, a una filosofía que postula el diálogo, la comunicación, como procedimiento de fundamentación y de consecución de acuerdos. Y finalmente, desde una teoría que sostiene en sus métodos la actitud del observador imparcial, "no-situado", abstraído del mundo, a una teoría que postula la actitud de la participación como presupuesto para la comprensión del sentido.

Habermas parece apuntar a la cuestión de la comprensión, en tanto el objeto de las ciencias sociales es una realidad pre-estructurada simbólicamente, pero parece hacerlo sólo en función de la comprensión del otro propia del científico social, o en dirección de una crítica social, sin aludir suficientemente a la necesidad de una comprensión de sí, o de una crítica de las ilusiones del sujeto en tanto desapropiación de sí como condición de posibilidad de un adecuado “acceso" al otro, y prolegómeno de una crítica social no distorsionada.

Desde el punto de vista de esta exigencia de una auto-comprensión y autocrítica, que creemos supuesta para la crítica social, nos resulta difícil concebir la pretensión de su teoría crítica como opuesta a la hermenéutica. Más bien, con Ricoeur, creemos que hermenéutica y crítica de las ideologías no pueden desconocerse mutuamente, antes bien, tienen mucho que aprender una de otra.

En este mismo sentido, no se comprende por qué han de presentarse como intereses contrapuestos el interés práctico de las ciencias histórico-hermenéuticas y el interés emancipatorio de las ciencias sociales críticas. En efecto, toda crítica necesariamente implica un momento de comprensión de la propia situación, de la propia tradición e historia cultural y personal, en suma, de la propia finitud. El primer engaño, la primera ceguera, la primera "ideología” es el desconocimiento de la propia finitud, del propio carácter de situado. Este desconocimiento nubla toda posible relación con lo otro y con los otros, y es capaz de deformar la relación con el mundo, y operar al modo de la indiferenciación mítica entre los mundos subjetivo, social y objetivo.

Por otra parte, el interés por la autonomía permanece abstracto si no va acompañado del reconocimiento de la dependencia sobre la que se recorta. Es errado asociar siempre la dependencia a la heteronomía. La autoridad puede ser autoritaria, pero no es sólo autoritaria. La paradoja de lo humano implica que la autoridad aliena pero también constituye. El Psicoanálisis podría dar cuenta de esta estructura originariamente ambigua de la subjetivación. Por lo tanto, ante la autoridad de la familia, de la cultura, de la tradición, deberá ponerse a la vez en juego la tarea de la comprensión apropiadora y la de la crítica desideologizadora. El movimiento crítico frente a los elementos ideológicos de la pertenencia, ha de recortarse sobre la pre-comprensión de la pertenencia, si quiere resultar verdaderamente liberador. En virtud de la pertenencia, el movimiento crítico deberá implicar un recorrido dialéctico de comprensión y explicación, en un proceso reiterado al infinito. De otro modo, el interés por la autonomía podrá caer en una nueva esclavitud: la abstracción de lo otro y del otro.

En tanto el lenguaje "nos baña”, el conocimiento de las distorsiones ideológicas, que se dan en el seno del lenguaje, no podrá ser nunca un conocimiento objetivante, pues no habrá nunca la distancia absoluta al objeto que tal conocimiento requiere. Desde Heidegger conocemos la correlación de Dasein y mundo. Desde esta perspectiva, la crí- 
tica de las ideologías no podrá descartar la hermenéutica, a la vez que la hermenéutica deberá despejar con más claridad el momento crítico necesario para completar la comprensión de sentido con la explicación que permita transformar las situaciones.

Consideramos este punto como uno de los análisis más agudos de Ricoeur en el artículo mencionado en el parágrafo anterior. En efecto, creemos que la crítica de las ideologías implica una crítica de las ilusiones del sujeto. Ninguna crítica de las ideologías es posible sin esta desapropiación de sí, esta distanciación de sí, ejemplificada en la hermenéutica del texto; desapropiación que requiere el reconocimiento de la propia finitud. Distanciación de sí y apropiación de la propuesta de mundo y de dimensiones nuevas de realidad abiertas por el texto, -o abiertas por la tradición, o abiertas por el otrovan de la mano. El reconocimiento de la propia finitud implica la conciencia de que quien critica es también criticable, está también situado, pertenece desde el principio a una cultura, a una historia social y subjetiva, no es nunca un sujeto abstracto más allá de toda condición. Habermas parece tener claro este concepto de sujeto y de razón, sin embargo, parece desconocerlo en su modo de oponer hermenéutica y crítica de las ideologías, interés práctico e interés emancipatorio. Incluso creemos que la crítica de las ilusiones del sujeto que es posible a partir del Psicoanálisis -y que no es sólo crítica sino también comprensión de sí- no ha sido asumida por la crítica de las ideologías con la consideración adecuada a la profundidad del aporte psicoanalítico y a las posibilidades infinitas que es capaz de abrir. Esta cuestión, sin embargo, la dejamos simplemente planteada, pues merecería el desarrollo de, al menos, un nuevo ensayo.

\begin{abstract}
Nos proponemos en este ensayo indagar en qué medida la crítica de las ideologías asume en sus pretensiones una de las principales conquistas de la racionalidad postmetafísica, a saber, el reconocimiento de la finitud del pensamiento y la existencia humana en general, particularmente en un aspecto específico: la propia situación, pertenencia o ser-en-el-mundo. En el fondo, la cuestión nos conduce a indagar a la vez los límites de la crítica de las ideologías y a preguntarnos sobre su necesidad de reconocimiento y diálogo con las pretensiones de la hermenéutica filosófica. Sin perder de vista su trasfondo común, intentaremos dilucidar un límite de la propuesta de la crítica de las ideologías respecto de esa asunción de la finitud de la existencia humana, y ver en ese límite una posibilidad de apertura a la hermenéutica y una exigencia de reconocimiento de sus pretensiones.
\end{abstract}

